Case Report

\title{
Exophiala jeanselmei causing subcutaneous phaeohyphomycosis in a healthy adult in Sri Lanka: A case report
}

\author{
PGRIS Welagedara ${ }^{1}$, LSM Sigera $^{1}$, IA Liyanage ${ }^{2}$, GMK Bogammana ${ }^{3}$, MM Abeykoon ${ }^{2}$, \\ PI Jayasekara ${ }^{1}$
}

Sri Lankan Journal of Infectious Diseases 2021Vol.11(1):47-51

DOI: http://dx.doi.org/10.4038/sljid.v11i1.8340

\begin{abstract}
Phaeohyphomycosis is a chronic infection caused by filamentous dematiaceous fungi and it embraces a wide range of clinical presentations. Subcutaneous phaeohyphomycosis is caused by traumatic implantation of aetiological fungi in subcutaneous tissue and is usually reported in agricultural workers in the tropics. Subcutaneous phaeohyphomycosis presents with various skin manifestations and is often misdiagnosed by clinicians. Exophiala sp is a common aetiological agent of post traumatic subcutaneous phaeohyphomycosis.

We present a middle aged farmer who presented with a subcutaneous nodule in his dominant hand. This swelling was initially diagnosed as a ganglion but turned out to be an abscess caused by Exophiala jeanselmei and the patient was cured by complete surgical excision without antifungal therapy. This case report aims to contribute to knowledge on this uncommon entity, in order to help practitioners to correctly diagnose and treat subcutaneous phaeohyphomycosis.
\end{abstract}

Keywords: Phaeohyphomycosis, Exophiala sp, Exophiala jeanselmei, subcutaneous phaeohyphomycosis, dematiaceous fungi

\section{Introduction}

Phaeohyphomycosis refers to a rare group of fungal infections caused by melanin producing (brown pigmented) moulds known as dematiaceous fungi. ${ }^{1}$ Melanin is a virulence factor of these fungi and its action is mainly by scavenging oxygen free radicals synthesized by phagocytes. ${ }^{2}$ Phaeohyphomycosis includes a spectrum of diseases ranging from localized cutaneous,

${ }^{1}$ Department of Mycology, Medical Research Institute, Colombo 8, Sri Lanka

${ }^{2}$ Department of Microbiology, Teaching Hospital, Kegalle, Sri Lanka

${ }^{3}$ Department of Surgery, Teaching Hospital, Kegalle, Sri Lanka

Address for correspondence: Dr Isanka Welagedara, Department of Mycology, Medical Research Institute,

Colombo 08, Sri Lanka Telephone:+94772004435 E-mail: isanka784@gmail.com

https://orcid.org/0000-0002-7341-8411

Received 23 December 2020 and revised version accepted 2 March 2021.

(c) () This an open-access article distributed under the terms of the Creative Commons Attribution License, which permits unrestricted use, distribution, and reproduction in any medium, provided the original author and source are credited. 
subcutaneous, deep, disseminated infections and allergic disorders of sinuses and lungs with varying morbidity. ${ }^{1}$ These moulds form septate hyphae, pseudo hyphae, yeast like cells or any combination of those in tissues. ${ }^{1}$ Apart from phaeohyphomycosis, dematiaceous fungi are capable of producing two other distinct forms of subcutaneous infections, named chromoblastomycosis and eumycetoma which are characterized by the formation of muriform bodies in the host tissues and identification of granules respectively. ${ }^{1}$

Species of Bipolaris, Curvularia, Exophiala, Exserohilum, and Wangiella are among the major aetiological agents of phaeohyphomycosis. ${ }^{2}$ Exophiala is a genus of black yeast like dematiaceous fungus with a worldwide distribution. ${ }^{1}$ It is commonly found in decaying wood and organic waste contaminated soil. ${ }^{1}$ Exophiala is a major aetiological agent of phaeohyphomycosis in both immunocompromised and immunocompetent individuals. ${ }^{1,3}$ E. jeanselmei complex is the most common species in the genus Exophiala to cause subcutaneous phaeohyphomycosis. ${ }^{1,4}$

\section{Case history}

A 59-year-old paddy farmer from a rural area of Sri Lanka presented with a painless lump over the dorsal aspect of his right wrist of two months' duration. He was unable to recall a significant history of preceding trauma to that particular site and the lump had gradually enlarged. The patient was immunocompetent with no co-morbidities.

On examination, the lump was well-defined, $2 \mathrm{~cm} \times 2 \mathrm{~cm}$ in size, non-tender with soft to firm consistency and slight fluctuance. It was not adhered to the underlying tissues and the overlying skin appeared normal without signs of inflammation. There was neither regional lymphadenopathy nor associated systemic symptoms. After a clinical diagnosis of a ganglion was made, the patient underwent excision of the lump under local anaesthesia. Surprisingly during surgery, pus was found within the lump. Sample of pus was sent to the Mycology Reference Laboratory at the Medical Research Institute for mycological investigation.

Direct microscopic examination of the specimen with $10 \% \mathrm{KOH}$ revealed brown pigmented branching septate fungal hyphae with rounded thick walled vesicles (Figure 1A). The rest of the specimen was inoculated into four bottles of Sabouraud Dextrose Agar (SDA) with chloramphenicol for culture and incubated at $37{ }^{\circ} \mathrm{C}$ and $26{ }^{\circ} \mathrm{C}$. After seven days of incubation, all four bottles of SDA yielded a pure growth of yeast like olivaceous black coloured small colonies. The colonies were initially slimy and skin like and the tease mount showed ellipsoidal, yeast-like, budding cells (Figure 1B). Growth of colonies at $37{ }^{\circ} \mathrm{C}$ was slower than that at $26{ }^{\circ} \mathrm{C}$. 

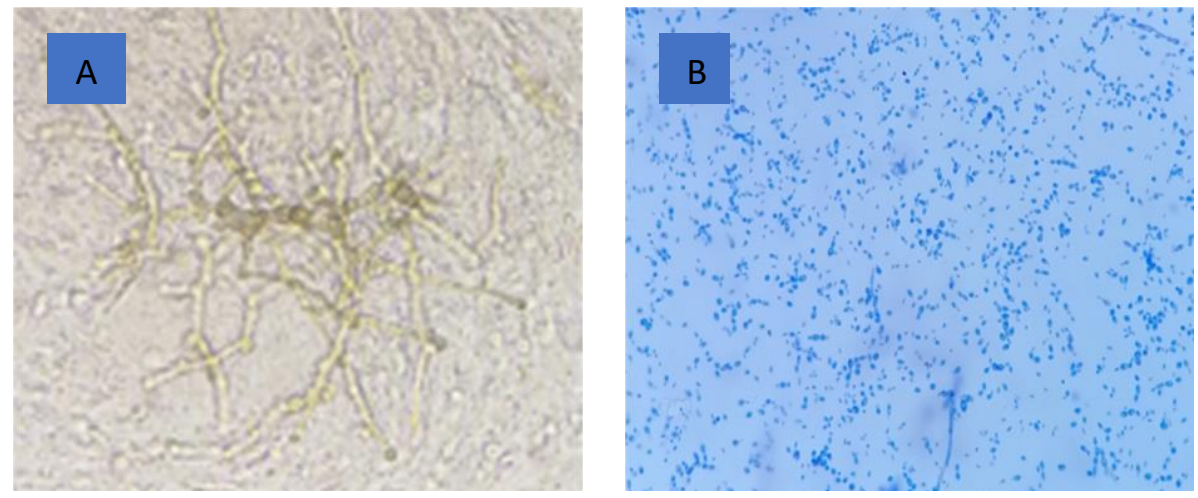

Figure 1

A: Direct smear showing brown pigmented, branching septate fungal hyphae with rounded thick walled vesicles (10\% $\mathrm{KOH}$ preparation $\mathrm{x} 400)$

B: Tease mount from a young colony with yeast cells, single or in short chains (Lactophenol cotton blue x 400)

Later, the colony became dome shaped with a velvety, suede-like texture and aerial mycelia developed (Figure 2A). The reverse of the colony was olivaceous-black in colour (Figure 2B). A tease mount preparation displayed fungal hyphae with conidia formed on the branches that arose laterally at right angles and apically (Figure $2 \mathrm{C}$ ). The conidiogenous cells were tubular, flask shaped with narrow, short annellated zones. The conidia were hyaline, smooth walled, oval, and collected in clusters around the conidiophore. There were no basal scars. Considering the macroscopic and microscopic morphology, the culture was identified as Exophiala jeanselmei.
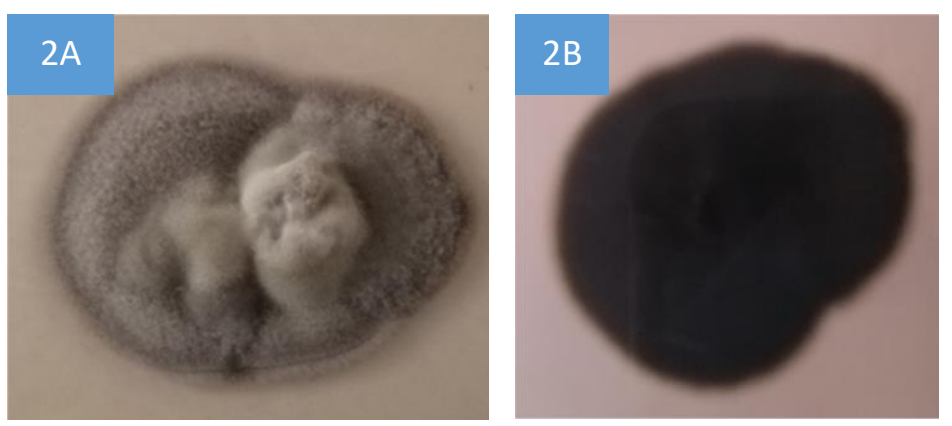

Figure 2

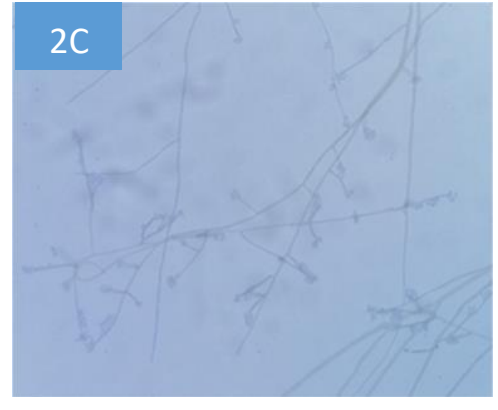

A: Colony on SDA after 3 weeks of incubation at $37{ }^{\circ} \mathrm{C}$ olivaceous black, velvety, with aerial mycelium

B: Reverse olivaceous black coloured

C: Slide culture showing septate conidiophores with clusters of conidia at the apex of long septate conidiophores (Lactophenol cotton blue x 400)

Since the patient was immunocompetent and was asymptomatic after surgical excision, he was not treated with antifungal drugs. He remained asymptomatic and was followed up at the surgical clinic. 


\section{Discussion}

This is a case of subcutaneous phaeohyphomycosis caused by Exophiala jeanselmei. A solitary nodule or cystic abscess at a site of a past injury are common presentations of subcutaneous phaeohyphomycosis as a result of subcutaneous inoculation of fungus contaminated materials. ${ }^{2}$ Males are affected more commonly than females due to their outdoor work, and the frequently affected age group is between 30 to 60 years. $^{2}$

According to the immune status of the patient, the presentation of subcutaneous infection may vary including cysts, papulonodules, abscesses, verrucous, pyogranuloma, hyperkeratosis, sinuses and non- healing ulcers, but the commonest manifestations are a solitary subcutaneous cyst or abscess as in our patient. ${ }^{2}$ Further, our case highlights the fact that fungal infections should also be included in the differential diagnosis patients presenting with a subcutaneous swelling as it may be misdiagnosed as lipoma, ganglion, fibroma, epidermal cyst or a foreign body reaction. ${ }^{2}$

The lesions are seen mainly on exposed areas of the body including fingers, wrist, toes, ankles and rarely on the scalp, face and neck. ${ }^{1,4}$ Our patient's lump was over the dorsum of his right wrist and although he could not recall, it's possible that he had a minor wound or abrasion with a contaminated material while he was working in the paddy field.

Subcutaneous phaeohyphomycosis may occur in immunocompetent individuals as in our patient. Similar case reports of otherwise healthy patients included a 68 -year-old female from UK with a palmer lump, a 46-year-old female from India complaining of a non-healing ulcer of the foot for 2 years and a 55-year-old Indian male with multiple lumps on his limbs. ${ }^{5-7}$

Management of subcutaneous phaeohyphomycosis comprises surgical excision with or without antifungal therapy. ${ }^{1,6}$ Antifungal therapy was not initiated in our patient since he was an immunocompetent person and complete excision of the small, solitary nodule was performed. Similarly, the 68-year-old healthy female from UK with a palmer lump and 55-year-old Indian male with multiple lumps were successfully managed with local excision without antifungal therapy. ${ }^{5-7}$

\section{Conclusion}

Subcutaneous phaeohyphomycosis presents with non-specific clinical features and the diagnosis requires laboratory identification. It is important that clinicians are aware of this entity and collect pus or tissue specimens for definitive diagnosis.

Conflicts of interest: There are no conflicts of interest.

Ethics statement: Consent was obtained from the patient for publication of this case report. Acknowledgement: We acknowledge the patient for consenting to publish the clinical details.

Funding source: None

Author contributions: PGRISW and LSMS carried out laboratory work for the identification of Exophiala jeanselmei from the cultured pus specimen. GMKB, IAL and MMA were involved with the clinical management of the patient and provided the patient information for the manuscript. PGRISW wrote the manuscript. PIJ supervised the laboratory procedures and manuscript writing. 


\section{References}

1. M. D. Richardson and D. W. Warnock. Fungal infection: diagnosis and management. Oxford: WileyBlackwell, 2012.

2. Chintagunta S, Arakkal G, Damarla SV, Vodapalli AK. Subcutaneous phaeohyphomycosis in an immunocompetent Individual: A case report. Indian Dermatology Online Journal. 2017; 8(1):29. doi: 10.4103/2229-5178.198770

3. "Exophiala | Mycology Online." https://mycology.adelaide.edu.au/descriptions/hyphomycetes/exophiala/ (accessed Nov. 21, 2020).

4. Y. A. Yoon, K. S. Park, J. H. Lee, et al. Subcutaneous phaeohyphomycosis caused by Exophiala salmonis, Ann Lab Med, 2012; 32(6):438-441 doi: 10.3343/alm.2012.32.6.438

5. Badali H, Chander J, Bayat M, et al. Multiple subcutaneous cysts due to Exophiala spinifera in an immunocompetent patient. Med Mycol. 2012; 50(2):207-13. doi: 10.3109/13693786.2011.603367

6. Borman AM, Fraser M, Schilling W, et al. Exophiala campbellii causing a subcutaneous palmar cyst in an otherwise healthy UK resident Medical Mycology Case Reports 2020; 29:43-45 doi: 10.1016/j.mmcr.2020.07.004

7. Mishra D, Singal M, Rodha MS, Subramanian A. Subcutaneous phaeohyphomycosis of foot in an immunocompetent host. Journal of Laboratory Physicians. 2011; 3(2):122.

doi:10.4103/0974-2727.86848 